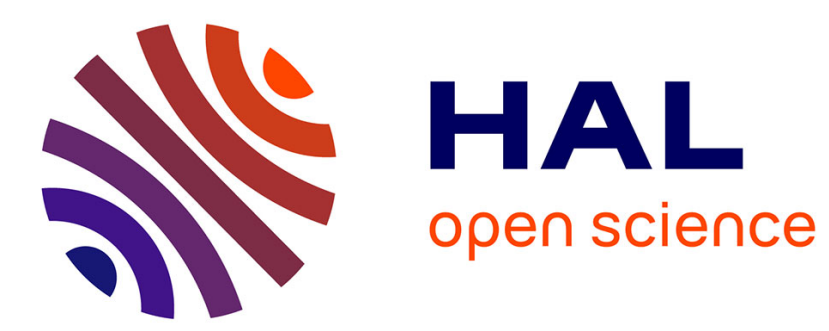

\title{
Use of a correlation chopper for time of flight neutron scattering - Part I: Theory of the deconvolution
}

\author{
J.L. Buevoz, G. Roult
}

\section{To cite this version:}

J.L. Buevoz, G. Roult. Use of a correlation chopper for time of flight neutron scattering Part I: Theory of the deconvolution. Revue de Physique Appliquée, 1977, 12 (4), pp.591-596. 10.1051/rphysap:01977001204059100 . jpa-00244213

\section{HAL Id: jpa-00244213 https://hal.science/jpa-00244213}

Submitted on 1 Jan 1977

HAL is a multi-disciplinary open access archive for the deposit and dissemination of scientific research documents, whether they are published or not. The documents may come from teaching and research institutions in France or abroad, or from public or private research centers.
L'archive ouverte pluridisciplinaire HAL, est destinée au dépôt et à la diffusion de documents scientifiques de niveau recherche, publiés ou non, émanant des établissements d'enseignement et de recherche français ou étrangers, des laboratoires publics ou privés. 
Classification

Physics Abstracts

7.114

\title{
USE OF A CORRELATION CHOPPER FOR TIME OF FLIGHT NEUTRON SCATTERING $\left(^{*}\right)$ PART I : THEORY OF THE DECONVOLUTION
}

\author{
J. L. BUEVOZ (**) and G. ROULT \\ Laboratoire de Diffraction Neutronique, Département de Recherche Fondamentale, \\ Centre d'Etudes Nucléaires de Grenoble, BP 85 Centre de Tri, \\ 38041 Grenoble Cedex, France
}

(Reçu le 28 juin 1976, accepté le 29 novembre 1976)

\begin{abstract}
Résumé. - Nous rappelons le principe de fonctionnement du chopper statistique et nous développons un formalisme matriciel qui permet de remplacer la déconvolution par la résolution d'un système de $N$ équations à $N$ inconnues. Nous considérons le cas d'une fonction signal rectangulaire idéale, et nous résolvons le système d'équations lorsque le nombre de canaux d'analyse est égal, ou supérieur, au nombre d'éléments de la séquence de fentes $\left\{a_{i}\right\}$. Nous montrons que, en pratique, le chopper statistique améliore la précision statistique, par rapport à un chopper à une fente, si la valeur moyenne de la fonction de diffraction est inférieure à la moitié du bruit de fond indépendant du temps. Nous montrons aussi que l'augmentation du nombre de canaux d'analyse, la durée de l'expérience restant constante, permet de mieux définir la forme des pics, sans modifier la précision relative de la mesure.
\end{abstract}

\begin{abstract}
We recall the principle of the correlation chopper and we develop a matrix formalism changing the deconvolution into the resolution of a linear system of $N$ equations with $N$ unknown roots. We consider the case of an ideal rectangular signal function and we solve the system when the number of analysing channels is equal to, or larger than the number of elements in the slot sequence $\left\{a_{i}\right\}$. We show that, in practice, the correlation chopper improves the statistical accuracy with respect to a one slot chopper if the mean value of the diffraction function is smaller than one half the time independent background. We show also that an increase of the number of analysing channels, the experiment lasting the same time, improves the definition of the shape of the peaks, without modification of the relative accuracy of the measurement.
\end{abstract}

1. Introduction. - In 1968, Gompf et al. [1] described a new kind of chopper. They called it a statistical chopper or correlation chopper. Its main advantage was to reduce the time independent background in the time of flight spectrum, without loss of statistical accuracy.

A great deal [2-11] has been written on this subject, using quite complicated mathematics. The experimentalist does not find a simple theory taking into account all the elements of the problem. Constructing a time of flight spectrometer for high pressure and high temperature studies, we have developed a matrix formalism allowing us easily to go from the ideal mathematical point of view to the experimental

(*) This work is a part of the thesis of J. L. Buevoz (1975), No CNRS AO 11644, Université de Grenoble, France.

$\left({ }^{* *}\right)$ Now at : Institut Laue-Langevin, 156X Centre de Tri, 38042 Grenoble Cedex. reality of a mechanical correlation chopper. In wha follows, we introduce the matrix formalism in the ideal case.

2. Principle. - Considering a chopper disc with one slot, the neutron pulse can be represented by the signal function $s(t)$, the diffraction function of the sample being $F(t)$.

The number of neutron counts detected during the time $\mathrm{d} t$ about the time $t$ is the convolution of $F(t)$ by $s(t)$ plus a time independent background $u$

$$
Z(t) \mathrm{d} t=\int_{0}^{T} F\left(t^{\prime}\right) s\left(t-t^{\prime}\right) \mathrm{d} t^{\prime} \mathrm{d} t+u \mathrm{~d} t
$$

$T$ is the chopper period.

The width of $s(t)$ is small compared to $T$ so :

$$
Z(t) \mathrm{d} t \simeq F(t) \mathrm{d} t+u \mathrm{~d} t .
$$

In the channel $k$ (width $\Delta t$ ) of the time of flight 
multichannel analyser, the number of neutrons is $Z_{k}$

$$
Z_{k}=\int_{(k-1 / 2) \Delta t}^{(k+1 / 2) \Delta t} Z(t) \mathrm{d} t
$$

and the expressions (1) and (2) become respectively, if $N$ is the total number of channels :

$$
\begin{aligned}
& Z_{k}=\sum_{i=0}^{N-1} F_{i} s_{k-i}+u_{k} \\
& Z_{k} \simeq F_{k}+u_{k} .
\end{aligned}
$$

The statistical accuracy of the measurement of the diffraction function is :

$$
\Delta F_{k}=\left(F_{k}+2 u_{k}\right)^{1 / 2}
$$

If there are $n$ slots, instead of one, on the disk, $Z(t)$ becomes the superposition of $n$ elementary spectra shifted in the same way as the slots on the disk. The mean flux on the sample is $n$ times higher, but the time independent background remains the same. If it is possible to deconvolute the function $Z(t)$, one finds a diffraction function of the same intensity as in eq. (5), but with a background $n$ times smaller.

Because two slots cannot overlap, it is easier to put them at distances at multiples of $\Delta T$ from each other. One has a new signal function $S(t)$

$$
S(t)=\sum_{i=0}^{N-1} a_{i} s(t+i \Delta t)
$$

where $s(t+i \Delta T)$ has the same shape as $s(t)$, but centered in $i \Delta T$ and $a_{i}$ is a coefficient whose value is +1 if there is a slot in $i \Delta T$ and 0 if there is no slot (the chopper is open or closed). The $a_{i}$ values define a sequence $\left\{a_{i}\right\}$ of $N$ elements.

The period of the signal function is $T=N \Delta T$. With this signal function, expression (1) becomes:

$$
\begin{array}{r}
Z(t) \mathrm{d} t=\sum_{i=0}^{N-1} a_{i} \int_{0}^{N \Delta T} F\left(t^{\prime}\right) s\left(t-t^{\prime}+i \Delta T\right) \times \\
\times \mathrm{d} t^{\prime} \mathrm{d} t+u \mathrm{~d} t
\end{array}
$$

and if we assume as a first approximation that $s(t)$ is a rectangular unit function of width $\Delta T$, then eq. (4) becomes :

$$
Z_{k}=\sum_{i=0}^{N-1} F_{i} a_{k-i}+u_{k} \quad(k=0 \ldots N-1)
$$

$F_{i}$ and $a_{k-i}$ being respectively the integrals of $F(t)$ and $S(t)$ in the interval $\Delta T$ abóut $(k-i) \Delta T$.

There are $N$ eq. (9) representing the counts in each of the $N$ channels of the multichannel analyser. Eq. (9) belongs to a linear system of $N$ equations. Following Weise [9] one can write the system in a matrix formalism

$$
Z=A F+U
$$

where $A$ is a $N . N$ circulant matrix :

$$
A=\left|\begin{array}{ccccc}
a_{0} & a_{N-1} & a_{N-2} & \ldots & a_{1} \\
a_{1} & a_{0} & a_{N-1} & \ldots & a_{2} \\
a_{2} & a_{1} & a_{0} & \ldots & a_{3} \\
\vdots & & & & \\
a_{N-1} & a_{N-2} & a_{N-3} & \ldots & a_{0}
\end{array}\right|
$$

One can solve the system if $\operatorname{det} A \neq 0$, in which case, there exists a matrix $B$ inverse of $A$ such that $B A=I$ ( $I=$ identity matrix). The matrix $B$ is also a circulant [18] that can be written in a similar way as $A$, defining a sequence $\left\{b_{i}\right\}$ inverse of $\left\{a_{i}\right\}$ :

$$
B=\left|\begin{array}{ccccc}
b_{0} & b_{1} & b_{2} & \ldots & b_{N-1} \\
b_{N-1} & b_{0} & b_{1} & \ldots & b_{N-2} \\
b_{N-2} & b_{N-1} & b_{0} & \ldots & \bar{b}_{N-3} \\
\vdots & & & & \\
b_{1} & b_{2} & b_{3} & \ldots & b_{0}
\end{array}\right| .
$$

The relation $B A=I$ reduces to a relation between the sequence $\left\{a_{i}\right\}$ and its inverse $\left\{b_{i}\right\}$

$\sum_{i=0}^{N-1} b_{i} a_{i-j}=\left\{\begin{array}{cl}+1 & \text { if } j=0 \\ 0 & \text { if } j \neq 0\end{array} \quad(j=0 \ldots N-1)\right.$

The solution for the linear system (10) is

$$
F=B(Z-U)
$$

the equation $k$ being :

$$
F_{k}=\sum_{i=0}^{N-1} b_{i-k}\left(Z_{i}-u_{i}\right) \quad(k=0 \ldots N-1) .
$$

The statistical accuracy of the measurement of $F_{k}$ is the square root of its standard deviation given by :

$$
\sigma^{2}\left(F_{k}\right)=\sum_{i=0}^{N-1}\left(b_{i-k}\right)^{2} \sigma^{2}\left(Z_{i}+u_{i}\right)
$$

$Z_{i}$ and $u_{i}$ are independent and satisfy Poisson's law thus :

$$
\sigma^{2}\left(F_{k}\right)=\sum_{i=0}^{N-1}\left(b_{i-k}\right)^{2}\left(Z_{i}+u_{i}\right) .
$$

A convenient sequence $\left\{a_{i}\right\}$ must satisfy relation (10) and minimize equation (17). One can find a complete study of the minimization problem in the text of Hossfeld and Amadori [6]. They introduce a relation between the number $N$ of elements in a sequence $\left\{a_{i}\right\}$ and the number $n$ of open slots

$$
\begin{gathered}
\left(a_{i}=+1\right) \\
r(N-1)=n(n-1) \quad(r \text { integer }) .
\end{gathered}
$$

This relation applies to all the possible sequences, and a general sequence $\left\{a_{i}\right\}$ is defined by the set of three parameters $(N, n, r)$. 
Another essential feature of the sequence $\left\{a_{i}\right\}$ is that its autocorrelation function is a Kronecker function :

$$
\sum_{i=0}^{N-1} a_{i} a_{i+j}=\left\{\begin{array}{cc}
n & \text { if } j=0 \\
r=\frac{n(n-1)}{N-1} & \text { if } j \neq 0
\end{array}\right.
$$

3. The deconvolution. - Let us consider an ideal case with a rectangular signal function and a general sequence $(N, n, r)$. In a first step we shall perform the deconvolution with a number of analysing channels equal to $N$, the number of elements in the sequence $\left\{a_{i}\right\}$, and calculate the statistical accuracy. Then we shall consider the special case of the binary feedback shift register sequences and compare the correlation chopper with a conventional one slot chopper.

Finally we shall look at what happens when one uses a number of channels equal to $m N$ ( $m$ integer) in order to obtain a better definition of the diffraction function.

In a further paper [13] we shall consider the experimental case of a triangular signal function and introduce the perturbations observed on the diffraction function when the signal function is produced by an imperfect mechanical device (mechanical chopper).

3. 1 DeConvolution IN AN IDEAL CASE WITH $(N, n, r)$ SEQUENCES. - Let us denote by $A^{*}$ the transposed matrix of $A$. From eq. (19) we can calculate the pro$\operatorname{duct} A \cdot A^{*}$.

$$
A A^{*}=A^{*} A=r E+(n-r) I
$$

$E$ is a $N . N$ matrix with all its elements equal to +1 and $I$ the identity matrix. With also have

$$
E=1 / n E A=1 / n A E \text {. }
$$

Introducing this value into eq. (20), we find :

$$
\left[\frac{r}{n(n-r)}\left(\frac{n}{r} A^{*}-E\right)\right] A=I \text {. }
$$

The matrix between the brackets is $B$, the inverse matrix of $A$, and taking $r$ into eq. (18)

$$
B=A^{-1}=1 / n n-1 / N-n\left(\frac{N-1}{n-1} A^{*}-E\right) \text {. }
$$

The $b_{\mathbf{i}}$ element of the sequence $\left\{b_{\mathbf{i}}\right\}$ is

$$
b_{\mathrm{i}}=\frac{1}{n} \frac{N-1}{N-n} a_{\mathrm{i}}-\frac{1}{n} \frac{n-1}{N-n}
$$

when introducing eq. (24) into eq. (15), the result of the deconvolution is :

$F_{k}=\frac{1}{n} \frac{N-1}{N-n} \sum_{i=0}^{N-1} a_{i-k} Z_{i}-\frac{1}{n} \frac{n-1}{N-n} Z_{\mathrm{T}}-\frac{1}{n} u_{k}$

with

$$
Z_{\mathrm{T}}=\sum_{i=0}^{N-1} Z_{i}
$$

We see immediately that the time independent background is divided by $n$, the number of the chopper transmitting slots.

The term involving $Z_{\mathrm{T}}$ was called by Graffstein [10] the additional background and elsewhere background of ignorance. It appears here only when expressing $b_{i}$ in terms of $a_{i}$ (eq. (24)).

3.2 Statistical ACCURACY. - The calculation of the standard deviation of $F_{k}$ is performed in the Appendix. We find :

$$
\sigma^{2}\left(F_{k}\right)=\left(\alpha F_{k}+\beta Z_{\mathrm{T}}+\gamma u_{k}\right) .
$$

This expression is similar to those of the one slot chopper (eq. (6)) involving $F_{k}$ and $U_{k}$.

3.3 CASE OF A BINARY FEEDBACK SHIFT REGISTER SEQUENCE. - In such sequences (see for example Stern $\boldsymbol{e t}$ al. [15]) the number $N$ of sequence elements is $N=2^{P}-1$ and the number of transmitting slots $n$ equals

$$
(N+1) / 2 \text {. }
$$

The previous expressions (23), (24), (25) simplify and become respectively :

$$
B=A^{-1}=\frac{2}{N+1}\left(2 A^{*}-E\right)
$$

$b_{i}=\frac{2}{N+1}\left(2 a_{i}-1\right)= \pm \frac{2}{N+1}$

$F_{k}=\frac{4}{N+1} \sum_{i=0}^{N-1} a_{i-k} Z_{i}-\frac{2}{N+1} Z_{\mathrm{T}}-\frac{2}{N+1} u_{k}$.

The standard deviation of $F_{k}$ is now :

$$
\begin{aligned}
\sigma^{2}\left(F_{k}\right)=\frac{4}{(N+1)^{2}} \sum_{i=0}^{N-1}\left(Z_{i}+u_{i}\right)= \\
=\frac{4}{(N+1)^{2}}\left(Z_{\mathrm{T}}+N u_{k}\right) .
\end{aligned}
$$

If we calculate $Z_{\mathrm{T}}$ from eq. (9), setting

$$
\bar{F}=\frac{1}{N} \sum_{i=0}^{N-1} F_{i}
$$

for the mean value of the diffraction function, we find as a result :

$$
\sigma^{2}\left(F_{k}\right)=\frac{2 N}{N+1}\left(\bar{F}+\frac{4 u_{k}}{N+1}\right)
$$

In the case of a shift register sequence the statistical accuracy of the measurement is a constant over the whole diffraction pattern and proportional to the mean value $\bar{F}$ of the time dependent diffraction function.

4. Comparison with a conventional one slot chopper. - To compare these two kinds of choppers, we calculate the ratio $\gamma$ of their standard deviations

$$
\gamma=\frac{\sigma^{2}\left(F_{k}\right)_{\text {correlation }}}{\sigma^{2}\left(F_{k}\right)_{\text {conventional }}}
$$


If $\gamma<1$ the correlation chopper is better than the conventional one. For a thin chopper disc with horizontal axis, the standard deviation of $F_{k}$ is given in eq. (6), and for a Fermi type chopper (thick disc) the previous time independent background $u_{k}$ is divided by a factor $c$ representing its absorption of epithermal neutrons. For shift register sequences we have :

$$
\gamma=\frac{2 N}{N+1} \frac{\bar{F}+\frac{4 u_{k}}{N+1}}{F_{k}+2 \frac{u_{k}}{c}}
$$

Gompf [1] makes the comparison for a point $F_{k}=\bar{F}$ of the pattern. In this way a correlation chopper can be better than the conventional one for the top of large peaks, and worse for the small peaks. We prefer to make the comparison for a point $F_{k}=0$ of the time independent background, and so, if the correlation chopper is better, this is true for every point of the diffraction pattern. (Note that, in this case, the standard deviation for the conventional chopper is only $u_{k} / c$.)

In this condition, and if $N$ is large enough, one finds :

$$
\gamma \simeq 2\left(\frac{c \bar{F}}{u_{k}}+\frac{4 c}{N+1}\right) \leqslant 1
$$

or

$$
\bar{F} \leqslant\left(\frac{1}{2 c}-\frac{4}{N+1}\right) u_{k}
$$

The right hand side must be positive, so if a thick chopper disc gives a reduction of the epithermal flux by a factor $c>(N+1) / 8$ this chopper will always be better than a correlation chopper. If $c=1$ and $N$ is large, there is an advantage in using a correlation chopper whenever $\bar{F}<u_{k} / 2$.

To make a choice between correlation and conventional chopper, one must have an idea of the possible values for $u_{k}$ and $\bar{F}$. Thus, for elastic scattering on powdered samples, $F$ will be large because of the Maxwellian component in the diffraction function due to incoherent scattering in the sample. On the other hand, in inelastic scattering, the pattern will contain only a few peaks on a flat background and $\bar{F}$ will be small.

For our purpose : neutron scattering on powdered samples under high pressure or at high temperature, with the high epithermal flux of the Melusine light water reactor, we are very far away from a borderline case [12]. The correlation chopper was the only way for us to solve the problem of high time independent background.

Note: Writing $F=B(Z-U)$ means that a separate measurement of the background is performed. One cannot be sure to measure it exactly. So if this measurement is not performed, one must include in function $F$ a background equal to $2 u_{k} / N+1$.
Eq. (29) and (30) become

$$
F_{k}^{t}=\frac{4}{N+1} \sum_{i=0}^{N-1} a_{i-k} Z_{i}-\frac{2}{N+1} Z_{\mathrm{T}}
$$

and

$$
\sigma^{2}\left(F_{k}^{t}\right)=\frac{2 N}{N+1} \bar{F}^{t} \simeq 2 \bar{F}^{t}
$$

5. Deconvolution in the case of $\mathbf{m N}$ analysing channels ( $m$ integer ). - With $N$ analysing channels, if the resolution is quite good, only a few points will represent a diffraction peak. To improve the definition of its shape, one can use a sequence with $N$ large, but $N$ is limited by a compromise between machining limitations, resolution and luminosity. Narrow slots increase the resolution but the beam also must be narrow and this reduces the luminosity of the chopper. It is better to increase the number of analysing channels and thus the number of points defining the peaks. We shall only consider shift register sequences and no separate measurement of background.

A system of $\mathrm{mN}$ linear equations is substituted for system (10)

$$
Z=A^{\prime} F
$$

$A^{\prime}$ is a $m N . m N$ circulant matrix built from a sequence $\left\{a_{i}^{\prime}\right\}$ which is obtained by changing $a_{i}$ in :

- $m$ consecutive terms equal to $1 / m$ if $a_{i}=1$,

- $m$ consecutive terms equal to 0 if $a_{i}=0$.

We shall use the matrix $A=m A^{\prime}$ whose elements are 0 and +1 as in the previous section. Its transposed matrix is $A^{*}$.

Calculating the product $A^{*} \cdot A^{\prime}$, we find :

$$
A^{*} A^{\prime}=\frac{N+1}{4}(E+m K)
$$

where $E$ is a $m N \cdot m N$ matrix whose elements are all equal to 1 and $K$ is a circulant and symmetric $m N . m N$ matrix. Its first line is

$$
\begin{array}{rrrrrrrr}
\frac{1}{m^{2}} \mid m & m-1 & m-2 & \ldots & 2 & 1 & 0 & \ldots \\
& \ldots & 0 & 1 & 2 & \ldots & m-1 \mid .
\end{array}
$$

These terms are to a constant the autocorrelation of the sequence $\left\{a_{i}^{\prime}\right\}$ which is a triangle with width $\Delta T$. One obtains the other lines of matrix $K$ by consecutive circular permutations of one element. We always have $E=\frac{2}{N+1} E A^{\prime}$ so :

$$
\left[\frac{2}{m(N+1)}\left(2 A^{*}-E\right)\right] A^{\prime}=B A^{\prime}=K \text {. }
$$

The matrix $B$ is no longer the inverse of $A^{\prime}$, but calculating the product $B Z$, we find :

$$
F^{\prime}=B Z=\frac{2}{m(N+1)}\left(2 A^{*}-E\right) Z=K F
$$


and for the channel $k$ :

$$
\begin{array}{r}
F_{k}^{\prime}=\sum_{i=0}^{m N-1} K_{k-i} F_{i}=\frac{4}{m(N+1)} \sum_{i=0}^{m N-1} a_{i-k} Z_{i}- \\
-\frac{2}{m(N+1)} Z_{\mathrm{T}}
\end{array}
$$

The calculated function $F_{k}^{\prime}$ is the convolution of the diffraction function by the autocorrelation function of the sequence $\left\{a_{i}^{\prime}\right\}$. As the full width at half maximum (FWHM) of the function $K$ is $\Delta T$, much smaller than the period $T$, just as in the first section, we can say that $F_{k}^{\prime} \simeq F_{k}$.

The standard deviation of $F_{k}$ is :

$$
\sigma^{2}\left(F_{k}\right) \simeq \sigma^{2}\left(F_{k}^{\prime}\right) \simeq \frac{2}{m} \frac{N}{N+1} \bar{F}^{\prime} \simeq \frac{2}{m} \bar{F}
$$

(note that now a background $2 u_{k} / m(N+1)$ is contained in the diffraction function $F$ ).

6. Comparison of the statistical accuracy with different values of $\mathbf{m}$. - We shall suppose that the experiment lasts the same time whatever the value of $m$, so :

$$
\bar{F}(m=m)=\frac{1}{m} \bar{F}(m=1)
$$

and

$$
\sigma^{2} F_{k}(m=m)=\frac{1}{m^{2}} \sigma^{2} F_{k}(m=1)
$$

If we now compare the relative precisions $\Delta F / F$ we find :

$$
\frac{\Delta F_{k} / F_{k}(m=m)}{\Delta F_{k} / F_{k}(m=1)}=\frac{1}{m} \frac{F_{k}(m=1)}{F_{k}(m=m)} \simeq \frac{1}{m} m=1 .
$$

Although the standard deviations are improved by a factor $1 / \mathrm{m}^{2}$, the relative precision remains the same for all values of $m$. Note that the results for a conventional chopper is different. Indeed, as

$$
\begin{gathered}
F_{k}=\sqrt{F_{k}} \quad \text { and } F_{k}(m=m) \simeq \frac{1}{m} F_{k}(m=1) \text { then }, \\
\frac{\Delta F_{k} / F_{k}(m=m)}{\Delta F_{k} / F_{k}(m=1)}=\sqrt{m}
\end{gathered}
$$

There is a loss in statistical accuracy. When using a conventional chopper, one obtains the same result as with $N$ channels and an experiment $m$ times shorter.

7. Conclusion. - Owing to the matrix formalism which we introduce here, it is possible to solve easily the deconvolution problem. The result is exact with a rectangular signal function and as many analysing channels as elements in the sequence $\left\{a_{i}\right\}$. With $m N$ analysing channels ( $m$ integer) one obtains an approximate solution, using the same approximation as in conventional time of flight.

Our comparison of the standard deviation of the diffraction function is more restrictive than those of Gompf [1] but more realistic, because one is interested in the whole peak and not only in its top part. The background of ignorance appears only when introducing $\left\{a_{i}\right\}$ in the calculation instead of $\left\{b_{i}\right\}$. It seems not to play a special part in the problem.

To obtain a better definition of the shape of the peaks, it is necessary to increase the number of analysing channels. We show that for experiments lasting the same time, the relative precision of a measurement remains constant whatever the $m$ value. One can conclude that a large value of $m$ is necessary. This is not true when using a mechanical chopper, because increasing $m$ emphasizes the perturbations of the mechanical sequence (speed fluctuations, inaccuracy in machining, absorption variation...). In our experiment at the MELUSINE reactor, a value of 2 for $m$ seems to be very convenient $[12,14]$.

Appendix. - Standard deviation of $F_{k}$ When USing a general SeQuence $(N, n, r)$. - The standard deviation of $F_{k}$ is defined as :

$$
\sigma^{2}\left(F_{k}\right)=\sum_{i=0}^{N-1}\left(b_{i-k}\right)^{2}\left(Z_{i}+u_{i}\right)
$$

with

$$
b_{i-k}=\frac{1}{n} \frac{N-1}{N-n} a_{i-k}-\frac{1}{n} \frac{n-1}{N-n} .
$$

Introducing this value into (A.1) and developing the square, one finds :

$$
\sigma^{2}\left(F_{k}\right)=\left[\frac{n-1}{n(N-n)}\right]^{2} \sum_{i=0}^{N-1}\left[\left(\frac{N-1}{n-1}\right)^{2} a_{i-k}^{2}+1-2 \frac{N-1}{n-1} a_{i-k}\right]\left(Z_{i}+u_{i}\right)
$$


$a_{i}$ takes the values 0 or +1 , thus $a_{i}^{2}=a_{i}$ and :

$$
\begin{aligned}
\sigma^{2}\left(F_{k}\right)=\left[\frac{n-1}{n(N-n)}\right]^{2}\left\{\left[\left(\frac{N-1}{n-1}\right)^{2}-2\right.\right. & \left.\frac{N-1}{n-1}\right] \sum_{i=0}^{N-1} a_{i-k} Z_{i}+ \\
& \left.+\left[\left(\frac{N-1}{n-1}\right)^{2}-2 \frac{N-1}{n-1}\right]_{i=0}^{N-1} a_{i-k} u_{i}+\sum_{i=0}^{N-1} Z_{i}+\sum_{i=0}^{N-1} u_{i}\right\} .
\end{aligned}
$$

From eq. (25) we have

$$
\sum_{i=0}^{N-1} a_{i-k} Z_{i}=\left(F_{k}+\frac{1}{n} \frac{n-1}{N-n} Z_{\mathrm{T}}+\frac{1}{n} u_{k}\right) \frac{n(N-n)}{N-1}
$$

and we also have

$$
\begin{aligned}
& \sum_{i=0}^{N-1} a_{i-k} u_{i}=n u_{k} \\
& \sum_{i=0}^{N-1} Z_{i}=Z_{\mathrm{T}} \\
& \sum_{i=0}^{N-1} u_{i}=N u_{k} .
\end{aligned}
$$

Introducing these values into (A.4), the result can be written as follows :

$$
\sigma^{2}\left(F_{k}\right)=\left(\frac{1}{n} \frac{n-1}{N-n}\right)^{2}\left(\alpha^{\prime} F_{k}+\beta^{\prime} Z_{\mathrm{T}}+\gamma^{\prime} u_{k}\right)
$$

with

$$
\begin{aligned}
\alpha^{\prime} & =\frac{n(N-n)(N-2 n+1)}{(n-1)^{2}} \\
\beta^{\prime} & =\frac{(N-1)(N-2 n+1)}{(n-1)^{2}}+1 \\
\gamma^{\prime} & =\frac{(N-2 n+1)(n N-2 n+N)}{(n-1)^{2}} .
\end{aligned}
$$

\section{Bibliographie}

[1] GompF, F., Reichardt, W., Glaser, W. and Beckurtz, K. H., Neutron Inelastic Scattering, Vol. II Vienna (1968) 417.

[2] Galloway, R. B., Nucl. Instrum. Meth. 70 (1969) 262.

[3] VIRJo, A., Nucl. Instrum. Meth. 75 (1969) 77.

[4] Von JAN, R. and Scherm, R., Nucl. Instrum. Meth. 80 (1970) 69.

[5] WilHeIMI, G. and GoMPF, F., Nucl. Instrum. Meth. 81 (1970) 36.

[6] Hossfeld, F. and Amadori, R., Report Jül. 684 FF (1970).

[7] PRICE, D. L. and SköLD, K., Nucl. Instrum. Meth. 82 (1970) 208.

[8] Pellionisz, P., Nucl. Instrum. Meth. 92 (1971) 125.

[9] Weise, K., Nucl. Instrum. Meth. 98 (1972) 119.
[10] Graffstein, A., Nucl. Instrum. Meth. 119 (1974) 101.

[11] QUitTNER, G., Nucl. Instrum. Meth. 68 (1969) 290.

[12] Roult, G. and Bubvoz, J. L., A time of flight spectrometer for elastic neutron scattering on powdered samples (to be published).

[13] BueVoz, J. L. and Roult, G., Use of a correlation chopper for time of flight neutron scattering, Part II (to be published).

[14] Buevoz, J. L., Thèse, Grenoble, No CNRS : A011644 (1975).

[15] Stern, T. E., Cazemajou, J., Macherez, B., Valat, J. and Vignon, A., Rapport C. E. A. R. 2230 (1964).

[16] Berlekamp, E. R., Algebraic Coding Theory (MacGrawHill, Berlin) 1966. 